Lepr Rev (1988) 59, 193-194

\title{
Editorial
}

\section{AIDS AND LEPROSY}

AIDS is now a n accepted problem in certain leprosy endemic areas, particularly in Central Africa. Infection with HIV (Human Immunodeficiency Virus) leads to a profound drop in T-lymphocyte function. The effect of this is to lower resistance to a wide range of opportunistic and other infections. Among the infections that were recognized early as developing in individuals with AIDS were those with the Mycobacterium avium-intracellulare group. However, it was not long before it was realized that lowered resistance to $M$. tuberculosis itself was also a major problem especially in Central Africa. Tuberculosis in AIDS patients is also a problem in non-tropical areas. There has been not only a slowing down of the annual decline in total morbidity, but also, the suggestion of an upswing in the incidence of the disease in some areas.

With this in mind it is pertinent to ask a number of questions about the implications of the AIDS pandemic for leprosy workers. So far there have been few reports of a direct association between HIV infection and leprosy (Lamfers et al. $1987^{1}$ and a patient observed by Dr W R Freeman, Department of Ophthalmology, University of California and Dr T H Rea, Section of Dermatology, University of Southern California). However, it is necessary to remain vigilant because such an association may not have become more apparent for a number of reasons. The most important of these is that leprosy takes a long time to develop and patients may die from other causes resulting from HIV infection before leprosy becomes clinically apparent. On the other hand, leprosy might make a patient more susceptible to HIV as it is itself a disease with immunocompromising features. Moreover, there have been suggestions that tuberculosis may predispose an individual to HIV infection or may be the first clinical manifestation of HIV disease. The effect of further depression of host resistance to $M$. leprae in a patient who already has leprosy would be a swing across the spectrum - downgrading-towards the lepromatous pole. Thus, it is important to look for an increased incidence of multibacillary leprosy in an area where the disease is predominantly paucibacillary. Another possible way in which HIV infection could manifest itself is in a failure to respond to chemotherapy or to maintain progress after a course of chemotherapy. A defect in T-cell-mediated immunity without a concomitant defect in antibody function may result in erythema nodosum leprosum (ENL) being the first manifestation of recurrence of active leprosy in an immunosuppressed patient. Such a case has been described by Adu et al. ${ }^{2}$ following renal transplantation in a patient with latent leprosy. In this patient symptoms of ENL did not develop until 2 years after the onset of immunosuppressive therapy.

It has also been suggested that the effects of HIV infection in a leprosy endemic area might be quite unexpected, resulting in an increase in the detection rate of tuberculoid leprosy, if the effect is to turn subclinical leprosy into tuberculoid leprosy. Another unexpected finding might be an accelerated decline in the detection rate of leprosy if patients with lepromatous leprosy are unrecognized because they die before the onset of clinical symptoms. Thus, leprosy workers should be ready for any unexpected change in the existing pattern of disease.

At the present time, there is a need for more data on HIV levels in new patients presenting with 
leprosy in areas where there is a high incidence of HIV infection in the general population. It is important that parallel studies should be made on new patients presenting with tuberculosis and also on symptom free individuals in the general population.

An added problem exists in those areas where leprosy vaccine trials are being undertaken. Most of these vaccines contain live BCG, giving a possible risk of dissemination of the BCG organism. Many of the cases of dissemination of BCG in the world literature have been described in children with congenital immunodeficiency diseases. This suggests that there is a definite risk of dissemination of BCG in immunodeficiency states due to the HIV virus. Local reactions and dissemination of BCG have both been described in HIV positive individuals. Two were adults and five were children. Thus, BCG should not be used in HIV infected subjects with symptoms of immunodeficiency or lymphadenopathy. ${ }^{3}$ The World Health Organization and the Advisory Committee on Immunization Practices of the Unites States Public Health Service still recommend the continued administration of standard vaccines to symptomless HIV infected children. Moreover, there are some who believe that giving BCG before the onset of immunodeficiency might prevent the early onset of mycobacterial infection. Therefore, there appears to be no evidence so far to support a curtailment of leprosy vaccine trials among individuals who show no signs of clinical AIDS. However, vaccine should be withheld from patients with clinical evidence of disease. Those engaged in leprosy vaccine trials in HIV epidemic areas should be aware of the difficulties that will be encountered in assessing the results of these trials if the subjects are not HIV tested in parallel. It may be necessary to have HIV serology on all subjects that develop leprosy as well as a parallel sample of the surrounding population.

Those working in the leprosy field involved in HIV screening will have to concern themselves

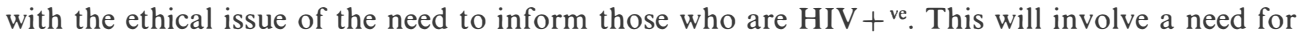
counselling with the added implicit expense. In addition, there will be a need for practical health education in parallel with the HIV screening.

J L TURK

Department of Pathology

Royal College of Surgeons of England

35/43 Lincoln's Inn Fields

London WC2A $3 P N$

National Institute for Medical Research

R J W REES

Mill Hill, London NW7 1AA

\section{References}

${ }^{1}$ Lamfers EJP, Bastiaans AH, Mravunac M, Rampen FHJ. Leprosy in the acquired immunodeficiency syndrome. Ann Int Med, 1987; 107: 111-12.

${ }^{2}$ Adu D, Evans DN, Millard PR, Calne RY, Shwe T, Jopling WH. Renal transplantation in leprosy. Brit Med J, 1973; 2: 280-1.

${ }^{3}$ Von Reyn CF, Clements, CJ, Mann JM. Human immunodeficiency virus infection and routine childhood immunisation. Lancet, 1987; ii: 669-72.

\section{WHO Guidelines for protection of personnel; HIV infection}

'Guidelines for personnel involved in collection of skin smears in leprosy control programmes for the prevention and control of possibleinfection with HIV' is a WHO document-WHO/CDS/LEP/ 87.1, issued recently, giving details of protection of personnel in areas where HIV infection is known or suspected to exist. The full text was printed in Leprosy Review, (1987) Number 3, 58, 207 and also appears in the International Journal of Leprosy, (1987) Number 2, 55, 421. 